\title{
The Behavior of Some Perfluorinated Analogs of Thenoyltrifluoroacetone in Aqueous Solution
}

\section{Supporting Information}

Maxim A. Lutoshkina,b, Ilya V. Taydakov and Boris N. Kuznetsov'b,d

aUniversité de Lyon, Université Claude Bernard Lyon 1, CNRS, UMR, 5256,

IRCELYON, Institut de recherches sur la catalyse et l'environnement de Lyon,

\author{
Villeurbanne, France
}

bInstitute of Chemistry and Chemical Technology SB RAS, Federal Research Center

“Krasnoyarsk Science Center SB RAS”, Krasnoyarsk, Russian Federation

CP. N. Lebedev Physical Institute of the Russian Academy of Sciences,

Moscow, Russian Federation 
dInstitute of Non-Ferrous Metals and Materials Science, Siberian Federal University,

Krasnoyarsk, Russian Federation

Table S1. The absorbance data for calculation of $\mathrm{pK}_{\mathrm{a}}(\lambda=341 \mathrm{~nm})$

\begin{tabular}{|c|c|c|c|}
\hline \multicolumn{2}{|c|}{$\mathrm{L} 1 ; \mathrm{C}(\mathrm{L} 1)=4.41 \cdot 10^{-5}$} & \multicolumn{2}{|c|}{$\mathrm{L} 2 ; \mathrm{C}(\mathrm{L} 2)=4.96 \cdot 10^{-5}$} \\
\hline $\mathrm{pH}$ & A & $\mathrm{pH}$ & A \\
\hline 7.20 & 0.931 & 7.20 & 1.031 \\
\hline 6.00 & 0.880 & 6.00 & 1.017 \\
\hline 5.60 & 0.816 & 5.60 & 0.978 \\
\hline 5.20 & 0.707 & 5.40 & 0.899 \\
\hline 5.00 & 0.622 & 5.20 & 0.849 \\
\hline 4.80 & 0.531 & 5.00 & 0.742 \\
\hline
\end{tabular}




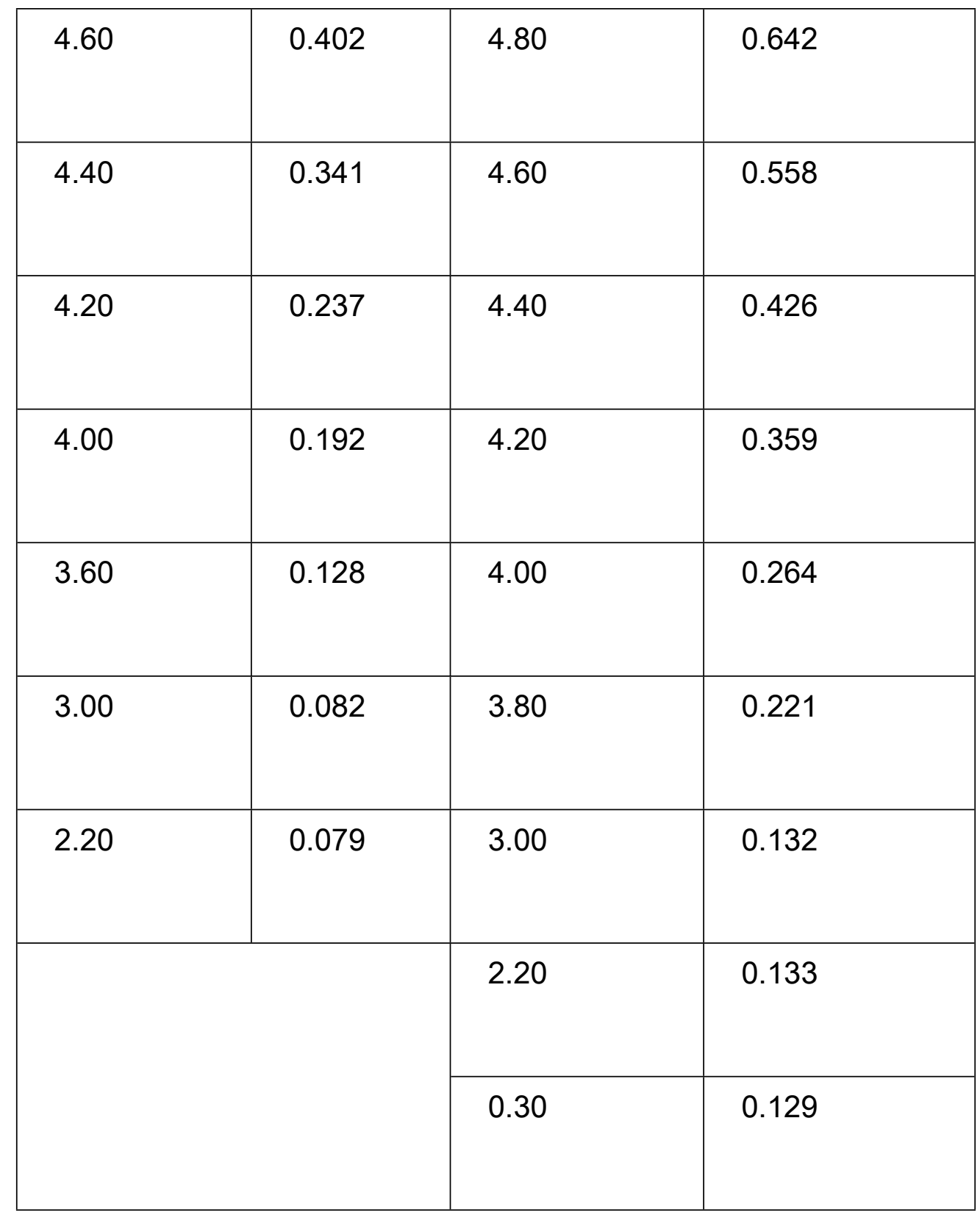

Table S2. The absorbance data for calculation of $\mathrm{pK}_{H}(\lambda=361 \mathrm{~nm})$ 


\begin{tabular}{|c|c|c|c|}
\hline \multicolumn{2}{|c|}{$\begin{array}{l}\mathrm{L} 1 \\
C(\mathrm{~L} 1)=5.88 \cdot 10^{-5}\end{array}$} & \multicolumn{2}{|c|}{$\begin{array}{l}\mathrm{L} 2 \\
C(\mathrm{~L} 1)=5.27 \cdot 10^{-5}\end{array}$} \\
\hline A & $\log (\mathrm{C}(\mathrm{HCl}))$ & A & $\log (\mathrm{C}(\mathrm{HCl}))$ \\
\hline 0.176 & 0.40 & 0.007 & -2.00 \\
\hline 0.200 & 0.50 & 0.117 & 0.40 \\
\hline 0.269 & 0.62 & 0.133 & 0.59 \\
\hline 0.303 & 0.67 & 0.197 & 0.72 \\
\hline 0.350 & 0.72 & 0.240 & 0.80 \\
\hline 0.359 & 0.76 & 0.277 & 0.85 \\
\hline 0.425 & 0.80 & 0.344 & 0.89 \\
\hline 0.481 & 0.85 & 0.417 & 0.96 \\
\hline 0.571 & 0.89 & 0.493 & 1.02 \\
\hline
\end{tabular}




\begin{tabular}{|l|l|l|l|}
\hline 0.646 & 0.93 & 0.552 & 1.09 \\
\hline 0.735 & 0.98 & \\
\hline 0.789 & 1.02 & \\
\hline 0.905 & 1.11 & \\
& & \\
\hline
\end{tabular}

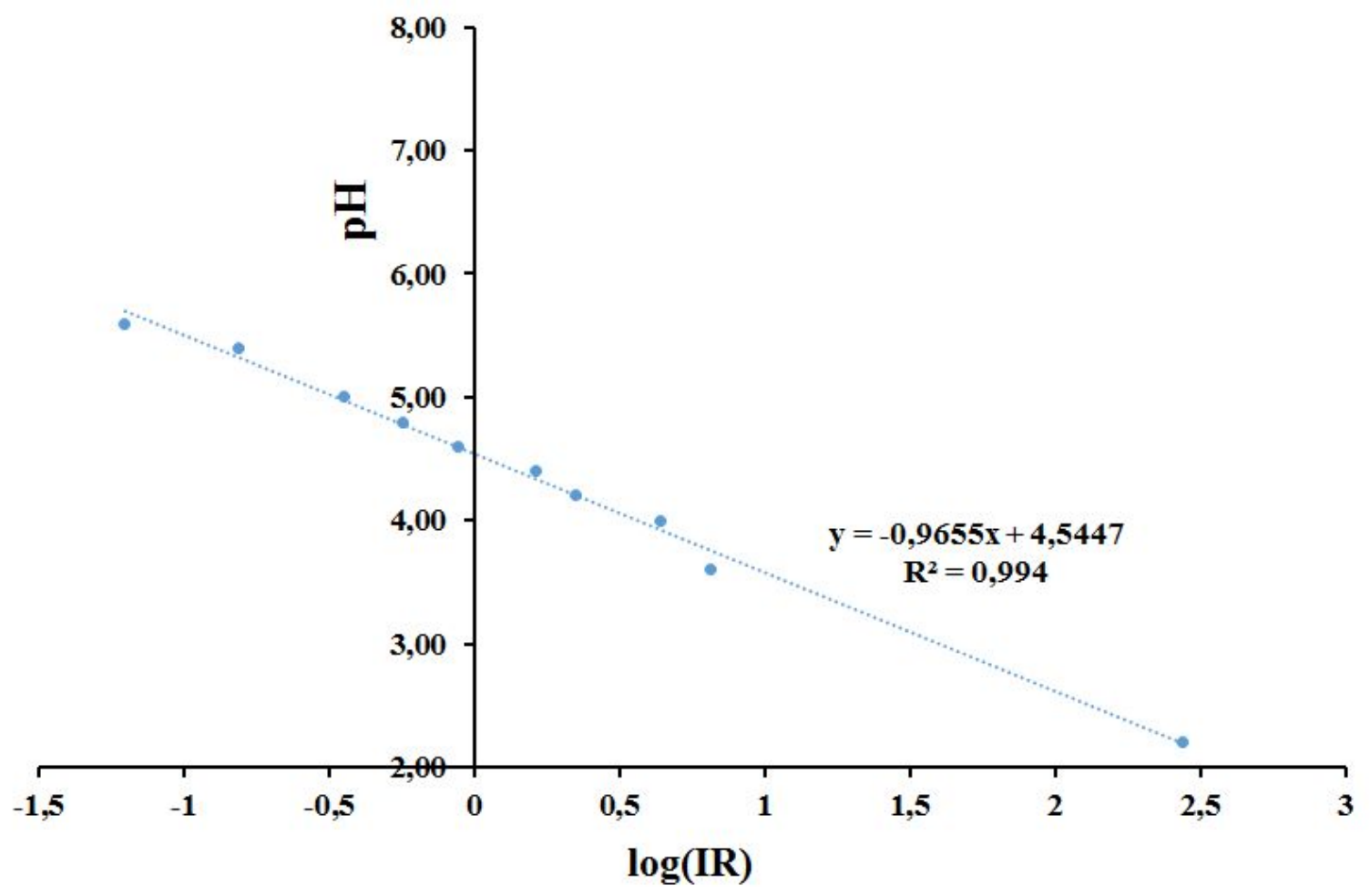

Figure S1. The $\log (\mathrm{IR})-\mathrm{pH}$ relationship for $\mathrm{L} 1$. 


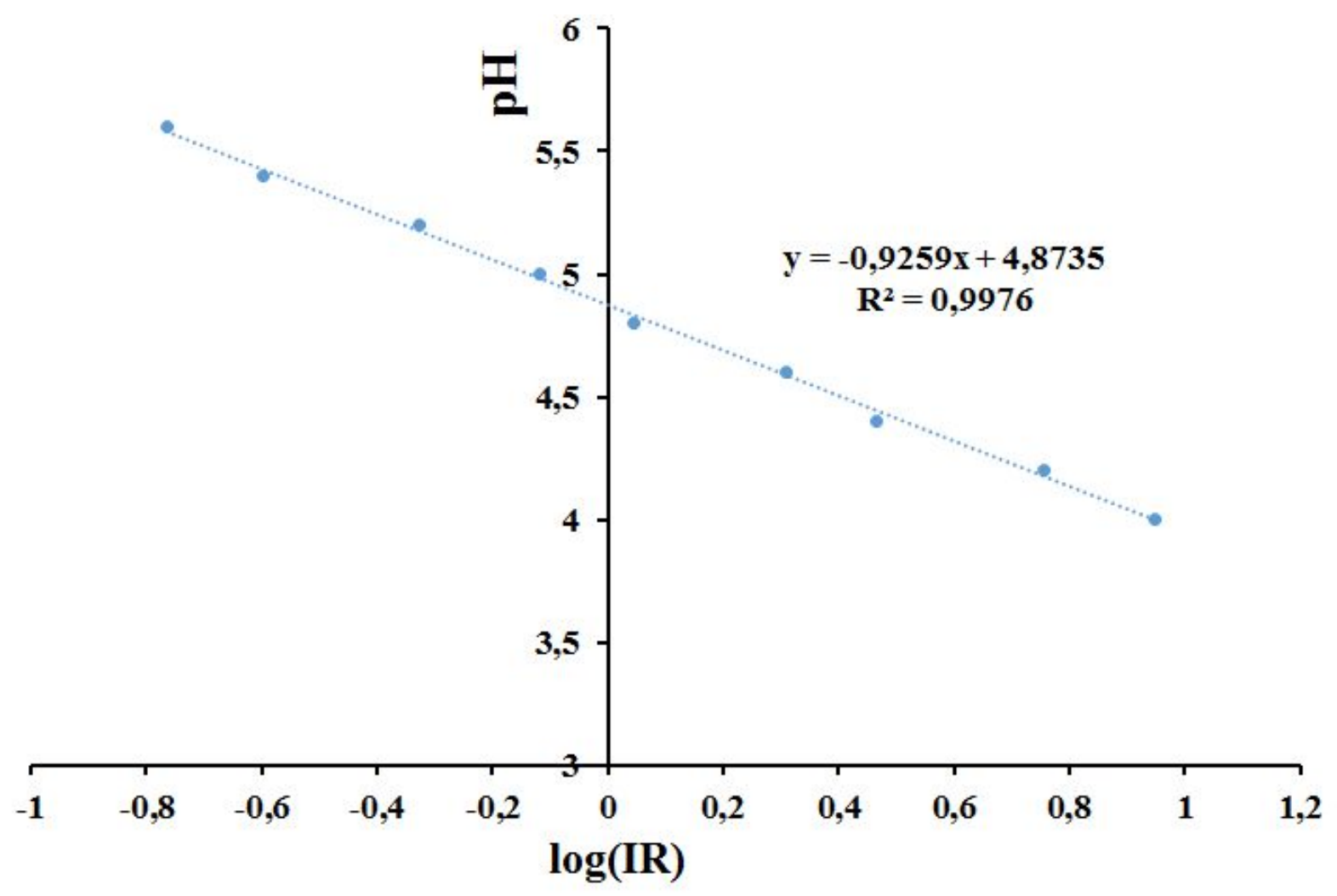

Figure S2. The log(IR)-pH relationship for $\mathrm{L} 2$.

NMR Data for L1:

${ }^{1} \mathrm{H}-\mathrm{NMR}(\mathrm{CDCl} 3,300 \mathrm{MHz}): \delta=14.99($ broad s, $1 \mathrm{H}, \mathrm{OH}), 7.87(\mathrm{~d}, 1 \mathrm{H}, \mathrm{J}=3.7 \mathrm{~Hz} \mathrm{CH})$,

$7.77(\mathrm{~d}, 1 \mathrm{H}, \mathrm{J}=4.8 \mathrm{~Hz}, \mathrm{CH}), 7.22(\mathrm{t}, 1 \mathrm{H}, \mathrm{J}=4.5 \mathrm{~Hz}, \mathrm{CH}), 6.52(\mathrm{~s}, 1 \mathrm{H}, \mathrm{CH}=\mathrm{C})$. 


\section{NMR Data for L2:}

${ }^{1} \mathrm{H}$ NMR $(\mathrm{CDCl} 3,300 \mathrm{MHz}): \delta=15.10($ broad s, $1 \mathrm{H}, \mathrm{OH}), 7.88(\mathrm{~m}, 1 \mathrm{H}, \mathrm{CH}), 7.79(\mathrm{~m}$, $1 \mathrm{H}, \mathrm{CH}), 7.23(\mathrm{~m}, 1 \mathrm{H}, \mathrm{CH}), 6.50(\mathrm{~s}, 1 \mathrm{H}, \mathrm{CH}=\mathrm{C})$.

Table S3. The absorbance data for Ce,Dy,Eu,Gd,Lu-L1 system at $350 \mathrm{~nm}, \mathrm{pH}=3.60$, $C(L 1)=3.70 \cdot 10^{-5} \mathrm{M}$

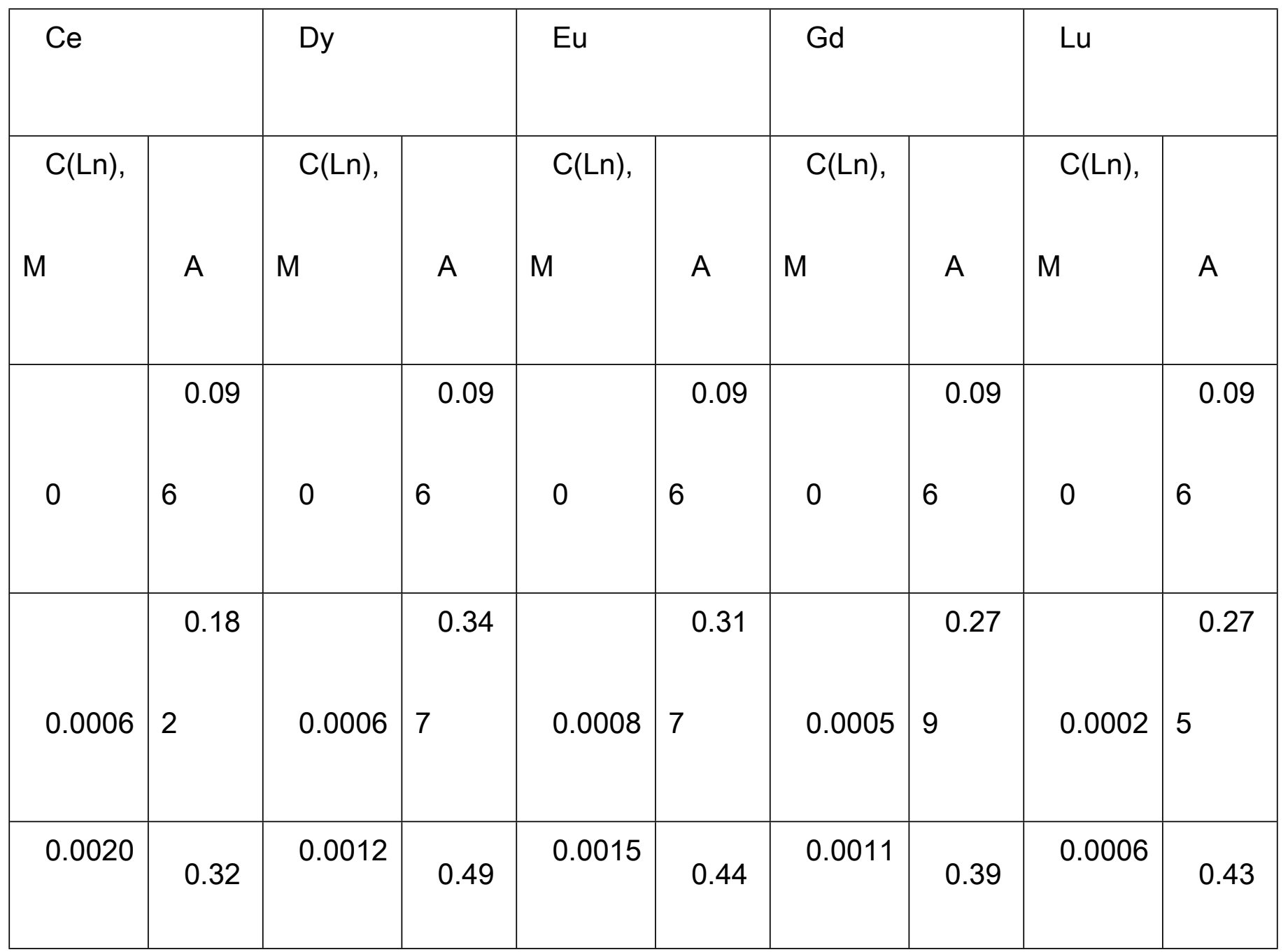




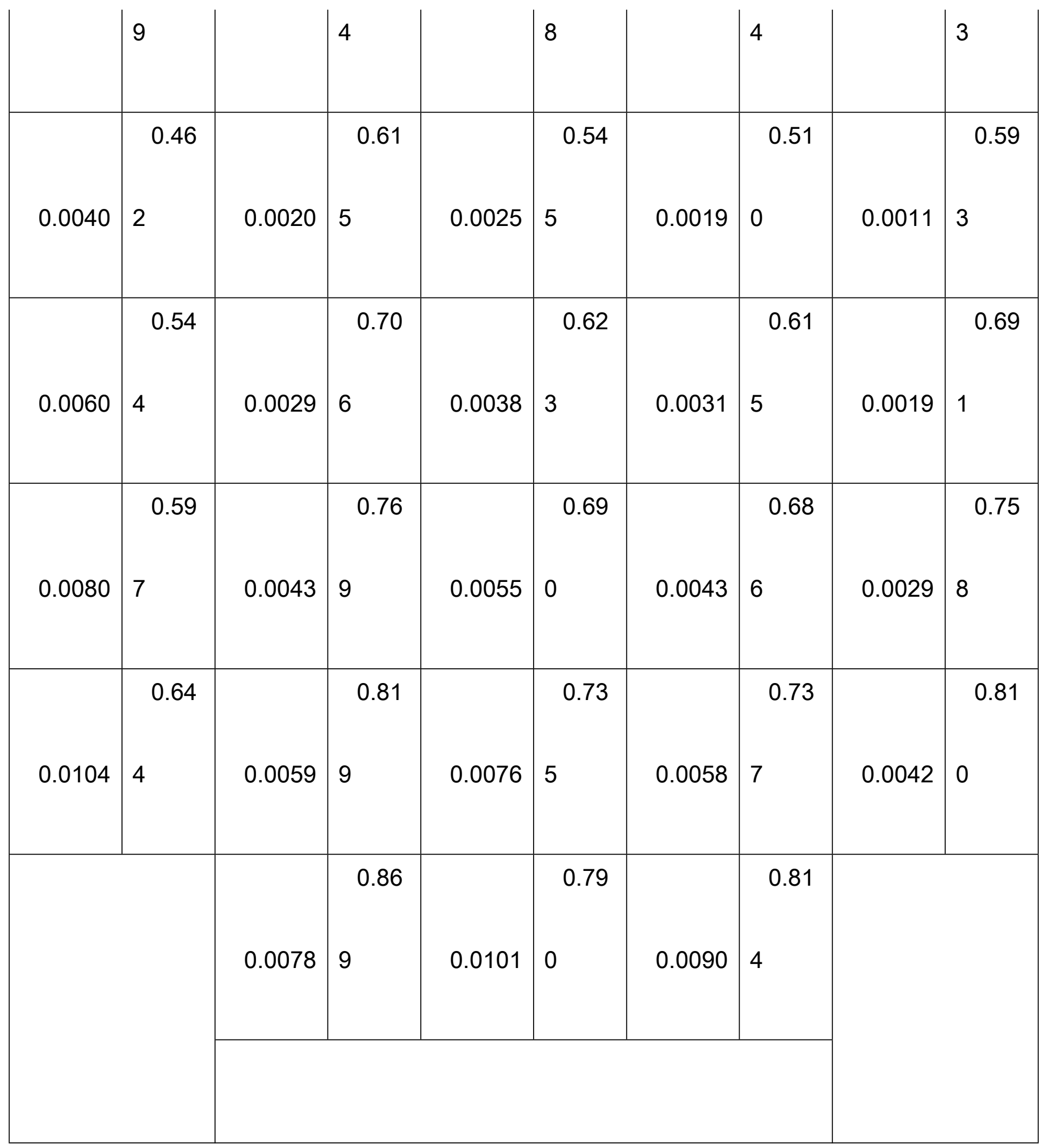


Table S4. The absorbance data for Er-L1 system at $350 \mathrm{~nm}$.

\begin{tabular}{|c|c|c|c|c|c|}
\hline \multicolumn{6}{|l|}{ Erbium } \\
\hline \multicolumn{2}{|c|}{$\begin{array}{l}\mathrm{C}(\mathrm{L} 1)=3.70 \cdot 10^{-5} \\
\mathrm{pH}=3.60\end{array}$} & \multicolumn{2}{|c|}{$\begin{array}{l}\mathrm{C}(\mathrm{L} 1)=3.70 \cdot 10^{-5} \\
\mathrm{pH}=3.20\end{array}$} & \multicolumn{2}{|c|}{$\begin{array}{l}\mathrm{C}(\mathrm{L} 1)=5.27 \cdot 10^{-5} \\
\mathrm{pH}=2.80\end{array}$} \\
\hline$C(L n), M$ & $A$ & $C(L n), M$ & A & $C(L n), M$ & A \\
\hline 0 & 0.096 & 0 & 0.096 & 0 & 0.102 \\
\hline 0.0002 & 0.255 & 0.0006 & 0.272 & 0.0012 & 0.355 \\
\hline 0.0006 & 0.391 & 0.0012 & 0.395 & 0.0022 & 0.494 \\
\hline 0.0012 & 0.551 & 0.0022 & 0.527 & 0.0034 & 0.629 \\
\hline 0.0022 & 0.673 & 0.0034 & 0.630 & 0.0049 & 0.718 \\
\hline
\end{tabular}




\begin{tabular}{|l|l|l|l|l|l|}
0.0034 & 0.752 & 0.0049 & 0.693 & 0.0065 & 0.821 \\
\hline 0.0049 & 0.804 & 0.0065 & 0.745 & 0.0081 & 0.878 \\
\hline 0.0081 & 0.876 & \multicolumn{3}{|l}{} \\
& & \multicolumn{3}{|l}{} \\
\hline
\end{tabular}

Table S5. The absorbance data for Nd,Pr,Sm,Tm,Yb-L1 system at $350 \mathrm{~nm}$

\begin{tabular}{|c|c|c|c|c|c|c|c|c|c|}
\hline \multicolumn{2}{|c|}{$\mathrm{Nd}$} & \multicolumn{2}{|c|}{$\mathrm{Pr}$} & \multicolumn{2}{|c|}{$\mathrm{Sm}$} & \multicolumn{2}{|c|}{$\mathrm{Tm}$} & \multicolumn{2}{|c|}{$\mathrm{Yb}$} \\
\hline $\begin{array}{r}C(\mathrm{~L} 1)= \\
\mathrm{pH}=\end{array}$ & $60 \cdot 10^{-5}$ & \multicolumn{8}{|c|}{$\begin{array}{c}C(L 1)=3.70 \cdot 10^{-5} \\
p H=3.60\end{array}$} \\
\hline$C(L n)$ & A & $\begin{array}{l}C(\operatorname{Ln}) \\
\mathrm{M}\end{array}$ & A & $\begin{array}{l}\mathrm{C}(\mathrm{Ln}), \\
\mathrm{M}\end{array}$ & A & $\begin{array}{l}\mathrm{C}(\mathrm{Ln}), \\
\mathrm{M}\end{array}$ & A & $\begin{array}{l}\mathrm{C}(\operatorname{Ln}), \\
\mathrm{M}\end{array}$ & A \\
\hline
\end{tabular}




\begin{tabular}{|c|c|c|c|c|c|c|c|c|c|}
\hline 0 & 0.13 & 0 & 0.096 & 0 & $\begin{array}{l}0.09 \\
6\end{array}$ & 0 & 0.09 & 0 & 0.09 \\
\hline 0.0006 & $\begin{array}{r}0.25 \\
4\end{array}$ & $\begin{array}{l}0.000 \\
6\end{array}$ & 0.174 & 0.0006 & $\begin{array}{l}0.26 \\
6\end{array}$ & 0.0003 & $\begin{array}{l}0.27 \\
2\end{array}$ & 0.0006 & $\begin{array}{r}0.32 \\
2\end{array}$ \\
\hline 0.0014 & $\begin{array}{l}0.36 \\
1\end{array}$ & $\begin{array}{r}0.001 \\
3\end{array}$ & 0.280 & 0.0013 & $\begin{array}{r}0.38 \\
5\end{array}$ & 0.0006 & $\begin{array}{l}0.39 \\
0\end{array}$ & 0.0011 & $\begin{array}{l}0.46 \\
9\end{array}$ \\
\hline 0.0024 & $\begin{array}{r}0.47 \\
7\end{array}$ & $\begin{array}{r}0.002 \\
5\end{array}$ & 0.376 & 0.0021 & $\begin{array}{l}0.49 \\
1\end{array}$ & 0.0010 & $\begin{array}{l}0.49 \\
4\end{array}$ & 0.0019 & $\begin{array}{l}0.57 \\
0\end{array}$ \\
\hline 0.0041 & 0.61 & $\begin{array}{r}0.003 \\
8\end{array}$ & 0.465 & 0.0031 & $\begin{array}{l}0.58 \\
0\end{array}$ & 0.0015 & $\begin{array}{r}0.60 \\
2\end{array}$ & 0.0029 & $\begin{array}{r}0.66 \\
5\end{array}$ \\
\hline 0.0061 & 0.76 & 0.005 & 0.556 & 0.0046 & $\begin{array}{r}0.64 \\
8\end{array}$ & 0.0022 & $\begin{array}{l}0.65 \\
6\end{array}$ & 0.0041 & $\begin{array}{r}0.73 \\
2\end{array}$ \\
\hline 0.0082 & 0.87 & 0.007 & 0.609 & 0.0063 & 0.70 & 0.0030 & 0.74 & 0.0056 & 0.79 \\
\hline
\end{tabular}




\begin{tabular}{|l|l|l|l|l|l|l|l|l|} 
& 7 & 6 & & 6 & & 1 & & 8 \\
\hline & 0.99 & & & 0.77 & & 0.78 & & 0.84 \\
\hline & 9 & & 0.0084 & 1 & 0.0040 & 5 & 0.0075 & 0 \\
\hline
\end{tabular}



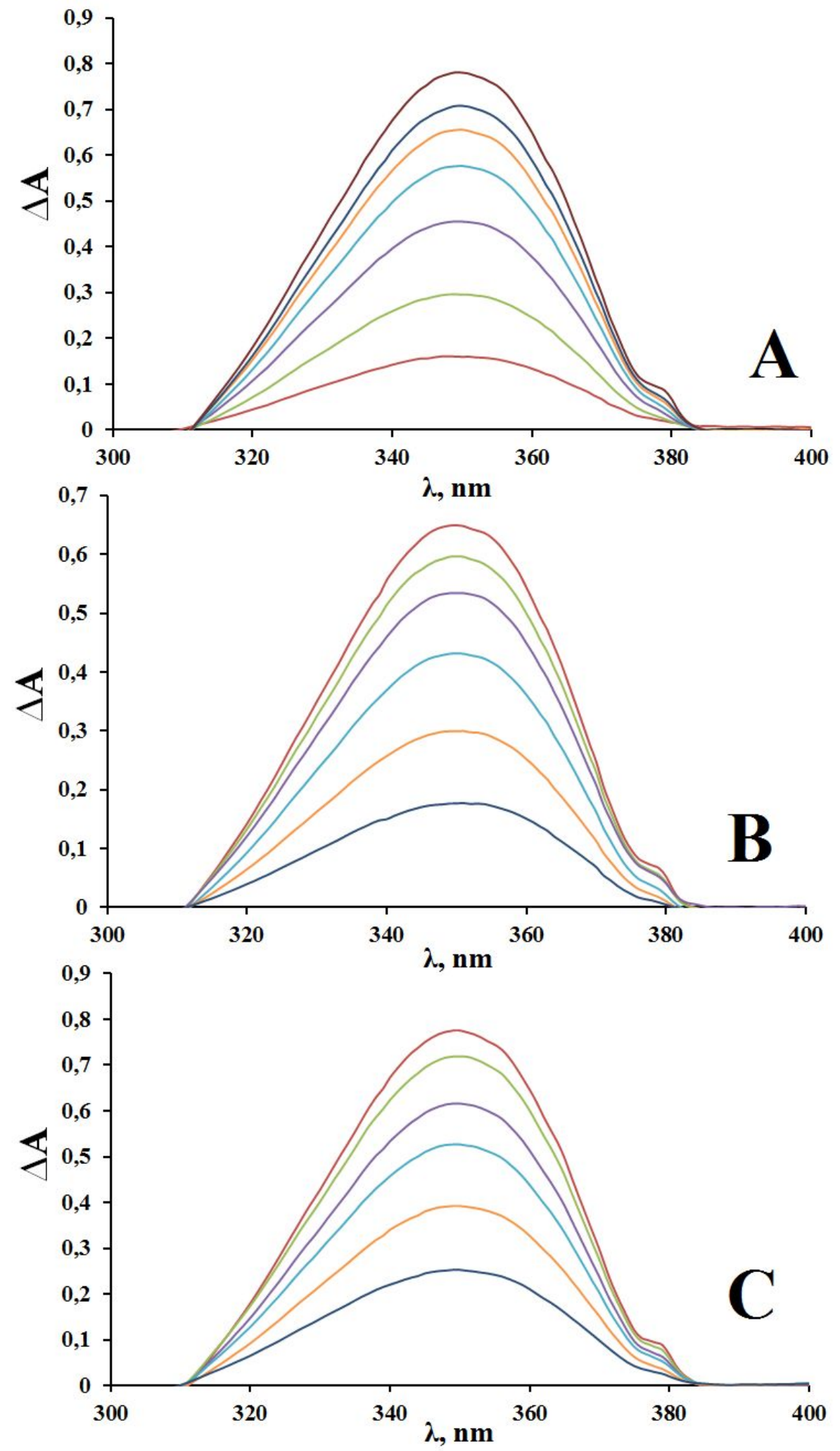
Figure S3. The $\Delta A-\lambda, n m$ curves for Er-L1 systems; pH: 3.60 (A); 3.20 (B); 2.80 (C).

Table S6. The logK values for calculation of $\alpha_{m}$ and $\alpha_{L}$

\begin{tabular}{|c|c|c|}
\hline $\operatorname{Ln}(I I I)$ & Gycine-Ln(III) & $\mathrm{OH}-\mathrm{Ln}(\mathrm{III})$ \\
\hline $\mathrm{Ce}$ & 3.4 & 8.65 \\
\hline $\mathrm{Pr}$ & 3.3 & 8.62 \\
\hline $\mathrm{Nd}$ & 3.3 & 8.49 \\
\hline Sm & 3.5 & 8.15 \\
\hline $\mathrm{Eu}$ & 3.5 & 8.06 \\
\hline $\mathrm{Gd}$ & 3.4 & 8.14 \\
\hline Dy & 3.6 & 7.89 \\
\hline $\mathrm{Er}$ & 3.7 & 7.82 \\
\hline $\mathrm{Tm}$ & 3.8 & 7.00 \\
\hline
\end{tabular}




\begin{tabular}{|c|c|c|}
\hline $\mathrm{Yb}$ & 3.9 & 7.55 \\
\hline $\mathrm{Lu}$ & 3.9 & 6.24 \\
\hline
\end{tabular}

